\title{
Stress Control Training for Pregnant Women: Applicability and Results
}

\author{
Eliana Aparecida Torrezan da Silva and Marilda Emmanuel Novaes Lipp* \\ Marilda Lipp Institute of Psychology and Stress Control Center, Brazil.
}

*Corresponding author: Marilda Emmanuel Novaes Lipp, Department of Psychology and Stress Control, Marilda Lipp Institute of Psychology and Stress Control Center, Brazil.

Received Date: November 24, 2019

Published Date: December 02, 2019

\section{Abstract}

Introduction: Stress is an ever-present topic in the most diverse areas of study.

Objective: the aim of the present study was to present data concerning the importance of prenatal intervention, in the context of the prevention of negative stress, and introduces strategies that may help to manage this stress, in pregnant women.

Method: For the stress control intervention, a protocol developed specifically for pregnant women was used.

Results: The data showed that the intervention reduced the level of stress and that it may have helped to provide a more comfortable birth for mother and child.

Conclusion: The study presented data and studies that support the possibility that pregnant women may have a psychological and medical gestational follow-up, and one that also includes the management of stress, whether preventive or by way of treatment.

Key words: Stress, Gestation; Pregnancy; Prenatal; Stress control; Prevention; Intervention; Stress control strategies

\section{Introduction}

The population in general suffers the consequences of day-today stress and strain and pregnant women who, besides the stress that is common to everyone else, also suffer worries, anxieties, fears arising from the pregnancy. Individuals who are unable to cope with these tensions elevate their level of stress, giving rise to the appearance and/or aggravation of various diseases.

A few theories described by Everly [1] address the dysfunction of the organism due to stress. The model referred to as "Hypokinetic Disease" [2] (hypo $=$ sub, kinetic $=$ movement), refers to a broad set of diseases resulting from the lack of a healthy expression/use of physiological mechanisms of response to stress. The absence of physical activity acts as a trigger for the emergence of pathologies and the pathogenic risk arises due to an overload on the target organ. Everly mentions that this model emphasizes the inadequate use of the body, promoting the concentration of energy in an organ, weakening it, and somatization occurs when confronted with the physiological reaction thus provoked. The homeostatic imbalance
[3] promoted by the stress reaction would be the elicitor of diseases. Everly proposes, initially, the stereotyping of responses which would be an individual tendency to exhibit psychophysiological reactive patterns to a variety of stressful stimuli. The second step refers to the frequent stimulation of stress, which promotes the failure in homeostatic recovery. The drain on the target organ thus favors pathological reactions.

The behavioral model proposes a theory of autonomic learning. This theory suggests that the appearance of psychosomatic manifestations is a result of frequent or prolonged reactions via stimulation of receptors. It also states that learned and biological factors interact to establish predisposing patterns of stimulation of the target-organ and that emotional, autonomous learning plays an important role in its arousal. The indication of the structure to be affected in the psychosomatic reaction will depend on the biological condition (genetic or environmental influences) and on the initial reactivity threshold and factors of learning, which affect this activation in an organism system. When the stimulation is 
excessively intense or chronic, the body will get weaker, becoming vulnerable to the appearance of disease. This approach agrees in part with the hypokinetic disease model [2] and homeostatic imbalance model [3] in respect of the physiological mechanism of response to stress, since the first-mentioned author asserts the lack of physical movement resulting in a physiological deficit, leaving the organism susceptible to stress, while the second-named specialist emphasizes the psychophysiological predisposition for some people to fall ill when faced with a stressful condition. However, for the behavioral model described by Lachman [4], it is also important to analyze the genetic makeup that may or may not favor stress reaction.

It must be emphasized that General Adaptation Syndrome [5] (GAS) has neuro-endocrine and endocrine changes as its reactive mechanism, particularly the adrenocorticotropic hormone, or ACTH, in moments of stress. Chronic maintenance of the resistance stage produces a drain on adaptive energy and a manifestation of the disease related to the stress occurs as a result of a reduction in the physical resources of stabilization and the subsequent exhaustion of the target-organ, which results from excessive stress. This process adjusts itself to the neural reaction [6] to explain the stress response.

The approach developed by Schwartz [7] suggests that when the environment (Stage 1) demands too much of an individual, the brain (Stage 2) performs the regulatory functions required to meet the specific demands. Depending on the nature of the demand of stress involved, body systems (Stage 3) will certainly be activated, while others may be simultaneously inhibited. However, if this process is maintained to the point that the tissue suffers deterioration or damage, the negative feedback loops (Stage 4) of the homeostatic mechanism will usually exert pressure on the brain to modify the directives to aid the afflicted organ (p.76). Accordingly, the negative feedback loops described by Schwartz dominate the normal physiological environment and are needed for effective, adaptive functioning. The author mentions that this deregulation mechanism, in the homeostatic processes in Stage 4, may lead to a series of stress-related diseases, through the heightened stimulation of the target organ. The shutdown of any feedback mechanism, from the system's point of view, is capable of leading to an imbalance and, therefore, disease. The final stage of this theory shares the premises of General Adaptation Syndrome [5] in which chronic stimulation reduces adaptive energy, promoting homeostatic dysfunction of the organism.

The "Disorders of Arousal" model [6] proposes a link between stress-related diseases, based on the phenomenon of limbic hypersensitivity. This limbic hypersensitivity phenomenon consists of a greater propensity for the activation of cognitive and affective efferent in the stress response. An excessively frequent activation of the target organ, either chronic or intense, due to limbic arousal, may exhaust it and impair adequate function. The authors rested on neural activation to explain the stress response, also previously maintained by Selye [5] and Schwartz [7].
It should be emphasized that the explanations cited for the stress response mechanism have one thing in common, namely the activation of the target organ. However, a variety of arguments are put forward for outlining the organism's reaction to stressful situations.

Note that the occurrence of stress may vary according to how much the individual was affected. Moderate stress triggers a movement of the organism towards action, so that it has the energy to cope with challenges. However, when its permanence is prolonged or its intensity great, the organism will need to expend a lot of energy, provoking an imbalance, making it potentially vulnerable to disease.

It is important to understand what emotional stress entails and thus be able to carry out a differential diagnosis with the aim of prevention and providing treatment. The diagnosis of stress must be conducted by a specialist. In more serious cases, a combination of medical and psychological treatment is recommended. As with the various symptoms of other problems, mainly cardiac diseases, it is advisable to perform a medical checkup in order to detect if any physical problems are involved. It is common in situations of physical impairment resulting from excessive or prolonged stress [12].

A recent study compiled data from stress-related areas of the brain, as well as the association with symptoms of depression. The study [13] suggests that depression may be viewed as an adaptation in order to conserve energy after a perceived loss of an investment in a vital resource, such as a relationship, group identity or personal asset. The tendency to process information in a negative way and experience strong biological reactions to stress (resulting from genes, trauma or both) may lead to dysfunctional beliefs about the self, the world and the future. These tendencies are mediated by alterations in the areas / networks of the brain involved in cognition and regulation of emotion. Depressive beliefs predispose individuals to make cognitive assessments that amplify perceptions of loss, normally in response to stressors that affect the available resources. These symptoms were presumably adaptive in our evolutionary history, however, are inadequate for contemporary times. So, as the study observes, when we think about a pregnant woman, this issue becomes relevant, as she is bringing a new human being into the world and it becomes important for her to maintain emotional balance and functional thinking, resulting in healthy, adaptive behavior.

It is important to remember that, when an individual is subjected to a high level of stress, a series of symptoms manifest themselves, both physical and psychological. Scholars [14] point to the following symptomatological condition of stress, asserting that the psychological symptoms are anxiety, tension, insomnia, alienation, interpersonal difficulties, self-doubt, excessive worrying, inability to concentrate on matters other than those related to the stressor, inability to relax, boredom, anger, depression, emotional hypersensitivity. Meanwhile, the physical symptoms include increased perspiration, stomach tension, hyperacidity of the 
stomach, muscle tension, tachycardia, hypertension, tightness of the jaw and grinding of the teeth, hyperactivity, cold hands due to constriction of the blood vessels and nausea. For these symptoms to occur, there must be a trigger.

Certain classes of the population have already been studied, for example children, freelancers, patients with cardiological, gastric or dermatological problems, among others, and pregnant women.

The study [15] involving Mexican-American women exhibited high rates of prenatal, maternal depressive symptoms in comparison with the general population. Though pregnant, acculturated Mexican-American women experience cultural stressors such as acculturation, acculturative stress and discrimination that may contribute to elevated depressive symptoms, the contribution of these socio-cultural correlates to depressive symptomology is unknown. Ninety-eight pregnant women of Mexican descent, in their first trimester, were recruited from a community hospital clinic. The women completed surveys about acculturation, acculturative stress, perceived discrimination, general perceived stress and maternal depressive symptoms as well as the potential protective factor of Mexican cultural values. Results showed that women who experienced greater acculturative and perceived stress, but not perceived discrimination or acculturation, reported a significantly high number of symptoms of depression during pregnancy. Also, women who experienced greater acculturative stress identified with a mixture of Mexican and American cultural values. However, only the Mexican cultural value of respect was protective against maternal depressive symptoms, while adhering to the Anglo values of independence and self-reliance was a risk factor. Results point to acculturative stress, over and above other cultural stressors, as a potential intervention target in culturally competent obstetric care. These findings have implications for maternal, mental health treatment during pregnancy, which likely affects maternal-fetal programming and may have a favorable effect on perinatal outcomes in the vulnerable Mexican-American population.

The study [16] conducted on pregnant women demonstrates the perception of expectant mothers about stress and introduces important information about the diversity of emotions perceived by these women. The aim of the study was to find out about the perceptions and experiences of expectant mothers in respect of stress in the pregnancy cycle, bearing in mind the magnitude of problems with stress in pregnancy, the high incidence and the troubling consequences, principally as a risk factor for postpartum depression. It was a qualitative and descriptive study, conducted in January 2018, on 10 pregnant women receiving prenatal care at a Family Development Center in a city in the Brazilian state of Ceará. Interviews were conducted using a semi-structured form, categorization of content according to Minayo (2012), resulting in four categories. The statements indicated that expectant mothers define stress as an event that results from certain situations that may occur in everyday life; that the pregnancy itself and their living conditions could cause them to experience stress. It may be concluded that the qualitative investigation enabled us to understand the perceptions and experiences of these pregnant women, and that the presence of emotional lability germane to this period, results in them experiencing disparate feelings.

Therefore, recognizing stress, its symptoms and its implications is necessary so that potential preventive measures can also be taken to avoid damage and harm, principally for the expectant mother.

Nowadays, women assume a wide range of different roles such as wife, professional, woman and mother, which suggests that they are constantly exposed to stress and its consequences. It is important to emphasize that the moment at which they are producing a child is important and therefore attention should be paid to the prophylaxis of the reaction of the organism during pregnancy. Being pregnant brings with it physical and emotional changes in the female body, changes in the family organization, in routine activities, giving rise to the manifestation of stressors.

Having covered this important issue since the 1980s, with various published studies [17], it inspired the authors to devise a procedure that could act upon prophylaxis and the treatment of stress.

For works relating to expectant mothers [8-11], the theoretical reference of limbic hyperstimulation was followed [6]. This choice is since the expectant mother is intimately connected with the fetus, with the cognitive and physiological connections being mutually active, and as a mother's emotional sensitivity relates to the fetus, so the stress response could perhaps affect both.

Stress Control Training (SCT) was developed by Dr. Marilda Lipp [18]. This training is based on principles of the cognitive-behavioral approach and aims at changing the individual's lifestyle, with an emphasis on improving quality of life in the following quadrants: social, affective, health and professional.

It is a brief focal treatment offering individuals the opportunity to recognize their limits, identify their stressors, familiarize themselves with the internal sources that generate stress and learn strategies for coping with the sources that generate tension, whether they be work-, family- or environment-related, or related to the characteristics of their personality. SCT emphasizes that it is not only work that generates stress but also the way individuals are and act in their everyday lives. The training comprises 12 to 15 sessions, on average.

Stress control training has been tested in a variety of experimental situations, on groups of office workers, police officers, patients with hypertension or psoriasis, amongst others. Since 1985, the effectiveness of SCT has also been effectively demonstrated in the clinical care of around 15,000 people in the Psychological Center for Stress Control (CPCS) [18].

SCT is not a technique nor an isolated procedure, but rather a set of procedures consisting of a functional analysis of the stressors and direct, objective action on what has been denoted as the four pillars of stress control: relaxation, diet, physical exercise and 
changes in the cognitive area. It employs several of the principles of stress inoculation training [18], of rational emotive therapy [20], as well as other useful behavioral techniques and procedures [17] in cases of stress, such as problem-solving techniques, assertiveness training, anxiety control training, instructions, management of time and the compulsion to hurry, relaxation, visualization exercises, identification and modification of thoughts that act as discriminative stimuli to trigger the stress response.

SCT aims to propose the following to those who perform it: to understand what stress is and identify the symptoms so that the individual can spot when tension overload is reaching a critical point; recognize the potential (internal and external) sources of stress and try to eliminate those which are capable of being changed; restructure their way of thinking and seeing the world so as to eliminate certain internal sources linked to their way of being; cope with anxiety in order to eliminate more of this internal source; to be assertive, without experiencing discomfort or becoming stressed out; to keep calm and resolve the problems of work and with the family without large increases in levels of stress; acquire stress management techniques to be used in unavoidable situations of stress; to use stress to their advantage to attain good levels of productivity and creativity; to recognize their limits and learn to respect them and establish priorities and improve quality of life in general.

Given this important stress management procedure, which has already been widely tested experimentally as well as clinically, the aim was to develop specific training for pregnant women.

The basis of stress control training for pregnant women (SCTP) [9], is Lipp's Stress Control Training [21], and may be classified, in part, within the proposals for assistance to expectant mothers [22], although it does affect other dimensions through which, specifically, the aim is to teach expectant mothers how to cope with their sources of stress. To this end, SCTP makes use of training sessions to inform, teach, instruct and encourage the use of techniques such as anxiety control, thought restructuring, relaxation, deep breathing, amongst other stress management strategies.

An earlier study by the author, [8] involving multiparous expectant mothers, compiled information on the level of stress and the symptoms, stressors and strategies used to cope with it. The results showed a high prevalence of stress in the sample and suggested that programs of prophylaxis and treatment of stress should be used with these women.

In another study [9], the effect of stress control on the outcome of the pregnancy was investigated, with women who had already given birth, multiparous women. A total of 30 pregnant women took part, aged between 19 and 34 (mean of 24.4 years); gestational age of between 16 and 32 weeks (mean of 22.1 weeks); average of 1.6 children; family income averaging 6.6 minimum salaries. These expectant mothers were divided into three groups of ten, in pre-partum. The initial testing evaluated stress, coping strategies and stressors as the characteristics of Type A behavior, irrational beliefs and sources of gestational stress. Stress Control Training
(SCT) for pregnant women was conducted in pre-partum with the experimental group (Stress Control Group - SCG). A control group (Discussion Group - DG) which attended a program of varied lectures that did not address psychological aspects, and the other control group (Time Passage Group - TPG), did not undergo any intervention. A total of 25 parturient mothers took part in the postpartum evaluation and the instruments evaluated stress, data on the newborn (NB) and the responses given by the mothers with regard to their feelings during the uterine contractions, at the birth, on seeing their child, and at the time of the first breastfeeding. The results showed that $100 \%$ of the pregnant women were situated in the stress resistance phase in pre-partum, with psychological symptoms being the most frequently detected. The stressors in pregnancy were the ones most frequently cited. The pregnant women were making use of an insufficient number of strategies to control sources of stress in pre-partum. The SCG demonstrated a significant reduction in stress post-partum, in comparison with the DG and TPG. Participants in the SCG had reduced symptoms of stress after the birth and showed less tension during uterine contractions. It was found that more women in the SCG were accompanied in the hospital, for the birth, by their husbands, suggesting the greater involvement of the latter in the pregnancy. The emotions shown by the mothers on seeing their babies and now of breastfeeding, suggested a feeling of happiness. Only three NBs (DG and TPG) were born prematurely and one was born underweight (SCG). The NB data indicated satisfactory birth conditions. It may be concluded that the SCT exerted an influence on the reduction of stress after the birth and in the pain resulting from the uterine contractions in the SCG.

Another study [23] was also concerned with studying strategies for diminishing discomfort during pregnancy and enhance the pregnant mother's wellbeing. The prospective cohort study with 71 low-risk, nulliparous expectant women, was distributed according to whether they took part (study; $n=38$ ) or not (control; $n=33$ ). The Multidisciplinary Program for the Preparedness for Birth and Maternity consisted of 10 appointments (between weeks 18 and 38), with educational, physical therapy and interactive activities. By means of a specific questionnaire, the following items were compared between the start and end of the program: occurrence (presence or absence), characteristics (location, type, intensity, frequency and duration) and the evolution of musculoskeletal discomfort. The outcome of the physical therapy techniques, in the program, was related to the reduction in intensity, frequency and duration and to the best possible evolution of musculoskeletal discomfort during pregnancy.

The research [24] mentions that the practice of relaxation may be an effective strategy for coping with stress during pregnancy, a stage of physical and emotional changes that requires adaptations. The objective was to evaluate the effects of the regular practice of a relaxation technique in pregnant women on emotional and physical variables: stress, anxiety, depression, heart rate (HR), and blood pressure (BP). Sample: 46 pregnant women, aged between 18 and 38, and living in Portugal. These women were divided into two 
groups, experimental and control. The experimental group received, between the $2^{\text {nd }}$ and $8^{\text {th }}$ month of pregnancy, a total of twenty to twenty-five individual sessions of visualization and progressive relaxation. To evaluate the effectiveness of the intervention, HR and BP were measured pre- and post-treatment, as well as anxiety, depression, and stress, using EADS-21. The results demonstrate the effectiveness of the intervention on the variables assessed.

Prenatal stress [25] (PS) has been linked to abnormal cognitive, behavioral and psychosocial outcomes in both animals and humans. Animal studies have clearly demonstrated PS effects on the offspring's brain, however, while it has been speculated that PS most likely affects the brains of exposed human fetuses as well, to date, no studies have yet examined this possibility prospectively, using an independent stressor (i.e. a stressful event over which the pregnant woman has no control, such as a natural disaster). The aim of this review is to summarize the existing animalrelated literature by focusing on specific regions of the brain that have been shown to be affected by PS, both macroscopically and microscopically. These regions include the hippocampus, amygdala, corpus callosum, anterior commissure, cerebral cortex, cerebellum and hypothalamus. We first discuss the mechanisms by which the effects of PS might occur. We show that maternal and fetal hypothalamic-pituitary-adrenal (HPA) axes and the placenta are the most likely candidates for these mechanisms. We can see that, although animal studies have obvious advantages over human studies, the incorporation of the findings with animals and the transfer of said findings to human populations remains a complex issue. Finally, we show how it is possible to circumvent these challenges by studying the effects of PS on brain development directly in humans, by taking advantage of natural or man-made disasters and prospectively assessing the impact and consequences of such stressful events on pregnant women and their offspring.

\section{Method}

\section{Subject}

The case of Y presents results from the application of the SCTP [9], showing that it can be of assistance, preventively, intervening as and when necessary.

Twenty-five-year-old patient Y, education - completed college; profession - commercial manager; socioeconomic level - middle class; married; first pregnancy.

The patient commenced treatment for SCTP at the start of her pregnancy at 10 weeks gestation. She did not use medication, except for vitamins, nor did she drink alcohol or smoke, or use any other illicit drugs. She did a prenatal course, had had no previous miscarriage/abortion and suffered no complications.

The patient started her SCTP therapy in 2016, complaining of high levels of anxiety, impulsiveness, immediacy, stress, difficulty in controlling her weight and dissatisfaction with her body image.

When she began her treatment, her main objective was to manage anxiety, stress and control of her weight, as she discovered she was pregnant.

\section{Instruments}

Psychological evaluation data: level of stress in resistance phase, prevalence of psychological symptoms according to Lipp's Inventory of Stress Symptoms for Adults (ISSL); minimum level of depression and anxiety according to the Beck Scale; few sources of stress resulting from family life; lack of assertiveness with a tendency to be more passive; sources of stress as a result of dysfunctional thoughts, type B behavior pattern as per classification [26], sources of gestational stress (Inventory of Sources of Gestational Sources, devised by Torrezan [8]); quality of life (Quality of Life Inventory, devised by Lipp) impaired with regard to her health; difficulty in developing and applying stress management strategies.

\section{Procedure}

The collected data were obtained via clinical interviews and the application of evaluation tools that are part of the battery of SCT tests [21]. Cognitive-behavioral techniques were used during treatment, together with the SCTP protocol [9].

Treatment was performed over 12 sessions in which aspects of Pregnancy, physical modifications accompanied by emotional changes were worked on; the relationship and differentiation between the symptoms of pregnancy and symptoms of stress; clarification about the level of stress and its symptoms; use of thought reformulation techniques to identify and reformulate dysfunctional ideals that were triggering internal stress; use of assertiveness training, employing behavioral testing, helping the patient to identify and develop verbal communication, so that she could express her thoughts and emotions; use of coping strategies to deal with anxiety emanating from external stressors; agenda reformulation, making time for herself; explanation of the four important areas of quality of life: social, affective, professional and health; the importance of changing habits for a healthy, balanced pregnancy; specific relaxation techniques for expectant mothers [27], and the use of mindfulness techniques focusing on the pregnant mother [28].

\section{Results and Discussion}

Patient data history: at 20 weeks gestation, the patient was already seeing an improvement in her level of stress and did not demonstrate significant rates of stress; she reduced her symptoms of stress; reduced anxiety and started using thought reformulation techniques to manage the sources of internal stress; she practiced relaxation for pregnant women at least once a week; she did not put on weight beyond what would be expected, in accordance with the medical checks performed during the prenatal period; she reformulated her diet and took care to eat in accordance with medical recommendation.

At 28 weeks, the patient had become capable of managing her reaction to the challenges she was facing, recognizing when the symptoms were beginning and already practicing coping strategies agreed in therapy sessions. She maintained an affective relation of encouragement and emotional support. She shared her ideas and emotions with her partner. She showed herself to be prepared for 
the baby's arrival, aware of what motherhood entailed, attempting to go through pregnancy joyfully and harmoniously.

The patient continued her treatment and attended her followups. The birth of her daughter occurred at 40 weeks, within the normal period of a term birth, and in healthy conditions.

This case considers and reinforces the positive results observed in other cases using Stress Control Training for Expectant Mothers already being cared for at the Stress Control Center clinic in the Brazilian city of São Paulo, and Marilda Lipp's Institute of Psychology and Stress Control Center, located in the city of Campinas, in the state of São Paulo, since 2000.

Stress control training has been used over the years in the most diverse areas of the population: children [29], teens and adults [30], as well as expectant mothers [31], who are producing new human beings and their emotional balance and control of stress is of the utmost importance.

\section{Conclusion}

The present study provides evidence in favor of stress control training for pregnant women to assist and complement the expectant mother's psychological monitoring, also helping in the prenatal period of pregnancy, aiming to take care of the mother and the development of her child in a harmonious, healthy manner, even in the face of stressful situations.

\section{Acknowledgement}

None.

\section{Conflict of Interest}

No conflict of interest.

\section{References}

1. Everly GS (1990) A Clinical Guide to the Treatment of the Human Stress Response. Plenum Press, New York, USA.

2. Kraus H, Raab W (1961) Hypokinetic disease. Charles C Thomas, Springfield, IL.

3. Sternbach R (1966) Principles of Psychophysiology. Academic Press, New York, USA.

4. Lachman SJ (1972) Psychosomatic disorders: A behavioristic interpretation. Wiley, New York, USA.

5. Selye H (1974) Stress without distress. Psychopathology of Human Adaptation, pp. 137-146.

6. Everly GS, Benson S (1989) Disorders of arousal and the relaxation response: speculations on the nature and treatment of stress-related diseases International Journal of Psychosomatics 36(1-4): 15-21.

7. Schwartz G (1977) Psychosomatic disorders and biofeedback. In: Maser J, Seligman M (Eds) Psychopathology. San Francisco: Freeman, USA.

8. Torrezan EA (1994) Stress and Pregnancy: Sources, Symptoms and Strategies. Dissertation [Master in Clinical Psychology] PUC-Campinas.

9. Torrezan EA (1999) The Effect of Stress Control on Pregnancy Outcomes. Thesis [Doctorate in Psychology and Profession] PUC-Campinas.

10. Torrezan-Silva EA (2001) Stress and its Implications for Child Development. Postdoctoral Report [Postdoctoral - Stress Laboratory PUC-Campinas and FAPESP] PUC-Campinas and FAPESP.
11. Torrezan-Silva EA (2013) Pregnancy and Preparation for Childbirth: intervention programs. World Health Magazine 37 (2): 208-215.

12. Lipp MEN, Tricoli V (2014) Interpersonal Relationship in the $21^{\text {st }}$ Century and Emotional Stress. Porto Alegre: Sinopsys.

13. Beck AT, Bredemeier K (2016) A Unified Model of Depression: Integrating Clinical, Cognitive, Biological, and Evolutionary Perspectives. Clinical Psychological Science 4(4).

14. Lipp MEN, Malagris LN, Novais LE (2007) Lifelong stress. Sao Paulo: Icon Publisher.

15. D’Anna-Hernandez KL, Aleman B, Flores AM (2015) Acculturative stress negatively impacts maternal depressive symptoms in Mexican-American Women during pregnancy. J Affect Disord 176: 35-42.

16. Monteiro PGA, Souza IC de, Rodrigues VC da C, Sena MM, Costa J da S, et al. (2018) Women's perceptions about stress experienced during pregnancy. Atlas-Qualitative Investigation in Health.

17. Lipp MEN (1984) Stress and its implications. Psychology Studies 1(3-4): 5-19.

18. Marilda Lipp Institute of Psychology and Stress Control Center (IPCS) [Internet homepage]. Campinas, Sao Paulo, Brazil: Stress Online, Inc. 2016; [Quoted 2017 Feb 12].

19. Meichenbaum D (1985) Stress inoculation training. Plenum Press, New York, USA.

20. Ellis A (1973) Humanistic Psychology: the rational emotive approach. Juliam, New York, USA.

21. Lipp MEN (1989) Stress Control - Course Manual. Campinas, São Paulo: Psychological Center for Stress Control.

22. Read GD (1944) Childbirth without fear. Ed. Harper Brothers, London, UK.

23. De Conti MHS, Calderon IdeMParan, Consonni EB, Prevedel TTS, Dalbem I, et al. (2016) Effect of physiotherapy techniques on musculoskeletal discomforts of pregnancy. Rev Bras Gynecol Obstet 25(9): 647-654.

24. Bjorn MBN, Jesus SNde, Morales MIC (2013) Relaxation strategies during the gestation period. Health benefits. Relaxation strategies during pregnancy. Health benefits 24(2): 77-83.

25. Charil A, Laplante DP, Vaillancourt C, King S (2016) Prenatal stress and brain development. Brain Res Rev 65(1): 56-79.

26. Friedman M, Rosenmam RH (1974) Type A behavior and your heart. A Fawcett Crest Book, New York, USA.

27. Torrezan EA (1997) Relaxation for pregnant women. In Lipp MEN (Eds) Relaxation for All: Control your stress. Campinas: Papirus Publisher, pp 65-67.

28. Torrezan-Silva EA (2015) Wellness and Stress Prevention with Mindfullness Practice. Annals V International Stress Congress 46.

29. Bignoto M (2017) Preventive strategies applied to parents and their children in childhood and their outcomes in childhood. Symposium Cognitive Behavioral Interventions applied in the development of pregnancy, childhood, adolescence and the young adult. XI Brazilian Congress of Cognitive Therapies, Foz do Iguaçu, PR, Brazil 19-22.

30. Tricoli V (2017) Preventive Intervention Model applied in adolescence and its results in young adult. Symposium Cognitive Behavioral Interventions applied in the development of pregnancy, childhood, adolescence and the young adult. XI Brazilian Congress of Cognitive Therapies, Foz do Iguaçu, PR, Brazil 19-22.

31. Torrezan-Silva EA (2017) Preventive practices applied to maternal behavior and outcome in the first year of the child's life. Symposium Cognitive Behavioral Interventions applied in the development of pregnancy, childhood, adolescence and the young adult. XI Brazilian Congress of Cognitive Therapies, Foz do Iguaçu, PR, Brazil 19-22. 